\title{
A Matlab Tool for Tumor Localization in Parathyroid Sestamibi Scintigraphy
}

\author{
Marko Đurović, Milica M. Jankovic, Member, IEEE, and Ana Koljević Marković
}

\begin{abstract}
Submarine method for localization of parathyroid tumors (PT) has proved to be effective in case of typical pitfalls of conventional scintigraphic methods (combined subtraction and double phase methods). It uses images obtained by standard dynamic parathyroid sestamibi scintigraphy suggested by European Association of Nuclear Medicine. This paper presents: 1) the developed Matlab interface that enables the implementation and evaluation of algorithms for the automatic application of Submarine method; 2) the algorithm for automatic extraction of the entire thyroid region from the background radioactivity using operations from mathematical morphology applied on dynamic scintigrams; 3 ) the results obtained by algorithm for localization and visualization of PTs based on estimation of exponential decreasing trend of time-activity curves. The algorithm was tested on a group of 20 patients with histopathologically proven PTs using developed Matlab interface.
\end{abstract}

Keywords - image segmentation, Matlab, parathyroid scintigraphy, Submarine method.

\section{INTRODUCTION}

$\mathrm{P}$ RIMARY hyperparathyroidism (PHPT) is an endocrine disorder that occurs due to adenoma (solitary or multiple), hyperplasia or cancer of parathyroid glands. Surgical removal of hypersecretion glands is the primary treatment for PHPT. Precise localization of the lesion is essential for minimally invasive intervention. Parathyroid scintigraphy is considered to be an important method of preoperative "imaging" that allows the localization of parathyroid tumors (PT) [1]-[2]. Conventional parathyroid scintigraphy methods include delayed scans technique, subtraction technique and tomoscintigraphy (SPECT,

Paper received October 9, 2015; revised October 26, 2015; accepted October 27, 2015. Date of publication November 15, 2015. The associate editor coordinating the review of this manuscript and approving it for publication was Prof. Irini Reljin.

This paper is a revised and expanded version of the paper presented at the 22th Telecommunications Forum TELFOR 2014.

This project is financially supported by the Ministry of Education, Science and Technological Development of the Republic of Serbia (no. 175016).

Marko Đurović, master student, is with the University of Belgrade School of Electrical Engineering, Bulevar kralja Aleksandra 73, 11120 Belgrade, Serbia (phone: 381-11-3218348; e-mail: marko_djurovic@rocketmail.com).

Milica M. Janković is with the University of Belgrade - School of Electrical Engineering, Bulevar kralja Aleksandra 73, 11120 Belgrade, Serbia (phone: 381-11-3218348; e-mail: piperski@etf.rs).

Ana Koljević Marković is with the Institute of Oncology and Radiology of Serbia, Pasterova 14, 11000 Belgrade, Serbia (phone: 38111-2067317, e-mail: md100218@gmail.com).
SPECT/CT) [2]-[4]. SPECT/CT technique in combination with ultrasound is superior to other scintigraphic techniques with a sensitivity of up to $95 \%$ [5], [6] However, due to the high cost of SPECT/CT devices clinical practice is oriented to the use of other parathyroid scintigraphic methods whose sensitivity varies from $50 \%$ to $86 \%$ [7], [8]. The most common failures of standard scintigraphic methods are in the case of hyperplasia, concomitant thyroid disorders and extremely small parathyroid lesions. Koljević et al. [9] presented Submarine method which uses images acquired by standard dynamic sestamibi scintigraphy, and proved to be effective in the mentioned critical cases. Submarine method is based on the observation of radioactivity changes in time in small regions of interest (ROIs) marked on parathyroid tissue to detect the characteristic short-term increase in radioactivity (10-30\%, duration 4-10 minutes) that occurs between the $10^{\text {th }}$ and $25^{\text {th }}$ minute (critical interval) from intravenous injection of radiopharmaceuticals, Fig. 1.

This paper includes three parts: 1) the presentation of the developed Matlab interface that enables the implementation and evaluation of algorithms for the automatic application of Submarine method; 2) the algorithm for automatic extraction of the entire thyroid region from the background radioactivity using operations from mathematical morphology applied on dynamic scintigrams; 3) the presentation of results obtained by algorithm for localization and visualization of PTs based on estimation of exponential decreasing trend of timeactivity curves (TACs, radioactivity changes in observed region in time) proposed in [10], [11]. The algorithm was tested on a group of 20 patients with histopathologically proven PTs using developed Matlab interface [12].

\section{METHOD}

\section{A. Acquisition protocol}

We have applied dual tracer double phase protocol for data acquisition suggested by European Association of Nuclear Medicine (EANM) [2]. This protocol includes the combination of subtraction protocol (if necessary, modified by injection of potassium perchlorate) and "delayed" scans protocol. Acquisition procedure includes the following steps: 1) intravenous injection of ${ }^{99 \mathrm{~m}} \mathrm{Tc}$ pertechnetate $(150 \mathrm{MBq} ; 4 \mathrm{mCi})$ in the butterfly vasofix braunule; 2) thyroid scan acquisition 20 minutes after injection of the ${ }^{99 \mathrm{~m}} \mathrm{Tc}$ pertechnetate $(128 \times 128,100 \mathrm{~K})$; 3 ) intravenous application of ${ }^{99 \mathrm{~m}} \mathrm{Tc}-\mathrm{MIBI}$ (500MBq, $13.5 \mathrm{mCi})$; 4) dynamic sestamibi acquisition that starts 
immediately after injection of ${ }^{99 \mathrm{~m}}$ Tc-MIBI (128x128, 35 minutes, 1 frame/min, zoom 3.2 , pixel size $1.5 \mathrm{~mm}$ ); 5) "delayed" scans of neck and mediastinum in oblique sections (position towards ultrasound localization of PT) 60 minutes after ${ }^{99 \mathrm{~m}} \mathrm{Tc}-\mathrm{MIBI}$ application (if necessary, this static acquisition is repeated until 120 minutes). In unclear cases, the entire acquisition procedure is repeated with the following modification: potassium perchlorate $\left(\mathrm{KClO}_{4}\right.$, $400 \mathrm{mg}$ ) was orally administrated before the intravenous injection of ${ }^{99 \mathrm{~m}}$ Tc-MIBI.

(a)

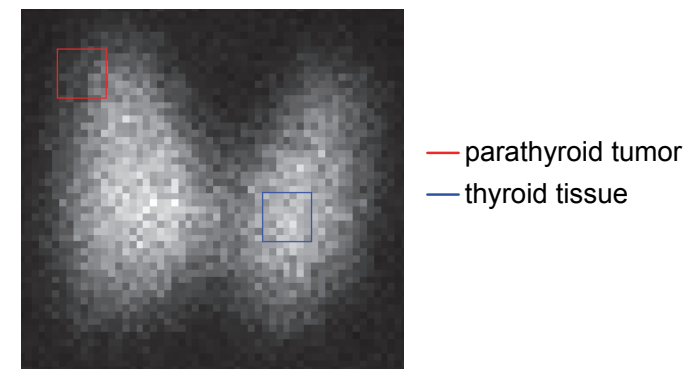

(b)

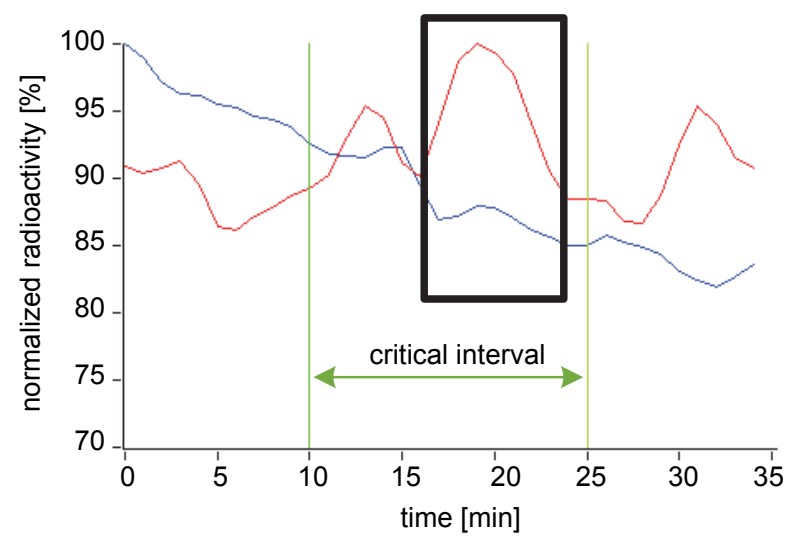

Fig. 1. Localization of parathyroid tumor (PT) using Submarine method (a) A single frame from a dynamic image sequence, taken at the $19^{\text {th }}$ minute, in a patient with PHPT (b) The radioactivity time-activity curve in the area

of PT shows a short-term increase compared to the exponentially decaying radioactivity in the contralateral thyroid region in the critical time interval (typical "peak" of radioactivity is marked).

For image acquisition, we used a digital gamma camera e.cam (Siemens $A G$, Erlangen, Germany) with a parallel hole, low energy, high resolution collimator.

The study was approved by the Ethics Committee. All patients signed a written informed consent form.

\section{B. Software specification}

Images were acquired and stored using Syngo e.soft 2007 software (Siemens $A G$, Erlangen, Germany). Conventional processing (subtraction analysis and observation of delayed scans) was performed using Syngo e.soft 2007 application. Semiautomatic Submarine analysis was performed in a specially designed Matlab (MathWorks Inc., Natick, Massachusetts, version R2013a) application.

\section{Matlab application}

Developed Matlab graphical user interface (GUI) provides the following steps:

- Loading DICOM file with a series of dynamic scintigrams (multiframe file)

- Cropping a rectangular ROI

- Morphological processing of a sum of dynamic scintigrams and automatic extraction of the thyroid region from the background (this step is applied on cropped scans, see section IID)

- Calculation and processing of radioactivity TACs for all small square ROIs within the automatically extracted thyroid region (the ROI size is selected by the user, for example $5 \times 5$ pixels). TAC processing includes normalization (to the TAC maximum) and cubic spline smoothing [13] (using Matlab function fit and SmoothingSpline method)

- Detection of "suspicious" ROIs (ROIs that potentially contain PT) according to the criterion that TAC doesn't have exponential decline, including a visual interpretation of the distribution of radioactivity in the suspicious area (using the correlation matrix of a suspicious ROI and surrounding ROIs, see section IIE).

D. Algorithm for the segmentation of dynamic scintigrams

The algorithm for the segmentation of dynamic scintigrams includes the following steps, Fig. 2:

- sum of all frames in one dynamic study, Fig. 2(a)

- edge detection of thyroid region (using Matlab function edge, method Canny [14]), Fig. 2(b)

- dilatation (using Matlab function imdilate), Fig. 2(c) adding pixels to the boundaries of objects in an image in order to obtain continuous edges of objects

- creating a binary image of edged region (using Matlab function imfill), Fig. 2(d)

- final extraction and drawing edges of the thyroid region on a binary image obtained in the previous step, (using Matlab function edge, method Canny), Fig. 2(e).

All methods for extracting edges (Sobel, Prewitt, Roberts, Laplacian of Gaussian, Zero-Cross and Canny) were tested using Matlab function edge. Fig. 3 shows the comparison of results of segmentation by different methods for edge extracting. Method Canny gives results that minimally differ from manually selected thyroid tissue, so this method was implemented in the application.

Matlab GUI provides additional manual settings of thresholds for Matlab functions edge (thresholds $T_{\text {Emin, }}$ $T_{\text {Emax }}$ ) and imdilate (threshold $T_{\mathrm{D}}$ ) in order to correct the edge of automatically extracted thyroid region.

\section{E. Algorithm for localization and visualization of $P T$}

Normalized and cubic spline smoothed TACs are fitted by exponential function $f(t)$ in the following form:

$$
f(t)=A e^{-k t}
$$

where $A$ is amplitude, $k$ is the speed of exponentional declining, and $t$ is time. Robust method with weight subfunction Bisquare, (within the Matlab function fit) is 
used for the implementation of fitting. The coefficient of determination ( $R$-Square), as a static measure of successful fitting, was calculated from samples of original and fitted curve.

(b)

(a)
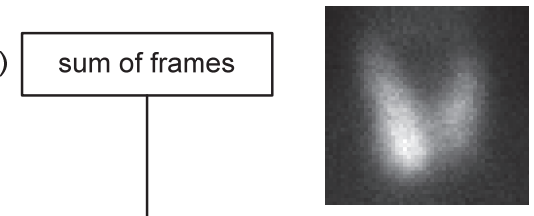

thyroid tissue edge detection

(c)

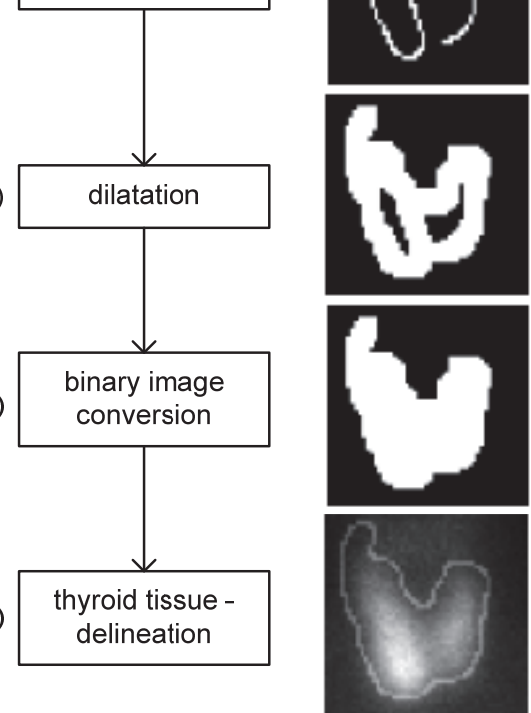

Fig. 2. Steps for automatic segmentation of a sum of all frames in a dynamic study. (a)

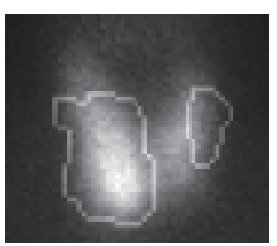

(c)

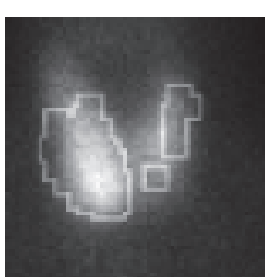

(e)

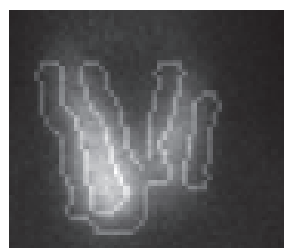

(b)

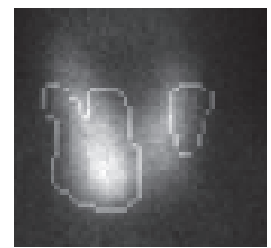

(d)

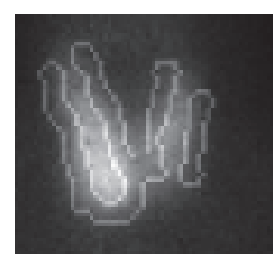

(f)

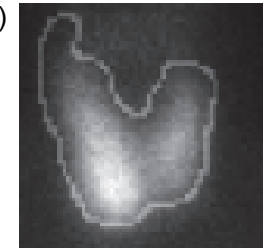

Fig. 3. Results of segmentation using different methods for extracting edges: (a) Sobel, (b) Prewitt, (c) Roberts,

(d) Laplacian of Gaussian, (e) Zero-Cross, (f) Canny method.

The visualization is based on the correlation method proposed in [10], [11]. For each "suspicious" ROI, the correlation matrix $C_{\mathrm{j}}$ is given in the following form:
$C_{j}=\left[\begin{array}{ccc}c_{-n-n} & \ldots & c_{+n-n} \\ \ldots & c_{00} & \ldots \\ c_{-n+n} & \ldots & c_{+n+n}\end{array}\right], \quad c_{00}=1, j=1, M$

where $M$ is a total number of "suspicious" ROIs, and $c_{x y}$ is the correlation between TAC corresponding to "suspicious" ROI (located at the origin $(0,0)$ ) and TACs for ROIs shifted for $x$-pixels along the $x$-axis and $y$-pixels along the $y$-axis in relation to the "suspicious" ROI, $-n$ $<x, y<n, n$ is the ROI size in pixels.

All ROIs which satisfy the condition $R$-square $<0.65$ (ROIs that do not have an exponential decay) and have a strong correlation (more than 0.95) with neighboring ROIs are considered as "suspicious" of PT and forwarded to a medical expert for a final decision. Mentioned threshold values are determined empirically. Matlab interface allows the correction of threshold values for R-Square and correlation.

\section{F. Patients}

The Matlab interface was tested on a randomly selected group of patients with PHPT, surgically treated at the Institute of Oncology and Radiology of Serbia in Belgrade. The group included 20 patients median age of 58 (38-73), 18 (90\%) female and two (10\%) male. All patients had an increased level of parathyroid hormone, median of 148 (80-456) pg/ml. Histopathology confirmed the presence of PTs in all patients, median PT volume $600(15-2250) \mathrm{mm}^{3}$. Also, $12 / 20$ patients underwent surgery of thyroid tumor.

\section{RESULTS AND DISCUSSION}

Table 1 presents the results of evaluation of the implemented algorithm for PT localization on a group of 20 patients with a histopathologically proven PT using Matlab interface shown in Fig. 4.

In the group of 20 patients, in $95 \%$ of cases $(19 / 20)$, the algorithm for the localization of parathyroid lesions correctly localized the lesion. In three patients, the algorithm additionally resulted in false positive locations that could be explained by patient movement (ID 4,7,15). In one patient with two parathyroid lesions, our algorithm found only one focus (ID 5).

Automatic algorithm for the segmentation of thyroid tissue presented in this paper has proved effective in the evaluated group of 20 patients. In $85 \%$ of cases it was not necessary to make the manual edge correction.

Automatic detection of thyroid tissue requires prior cropping the thyroid region by rectangular ROI, after which the algorithm determines precise edge detection of thyroid tissue within the selected rectangular ROI. Wide selection by rectangular ROI around the thyroid tissue may negatively affect the automatic detection of edges because the result of morphological operations may include other, neighboring part of the body where the radiopharmaceutical is retained. Preferably, a rectangular ROI needs to be selected so that the distance from the rectangular ROI and the "imaginary" edge of the thyroid tissue is less than 15 pixels. 


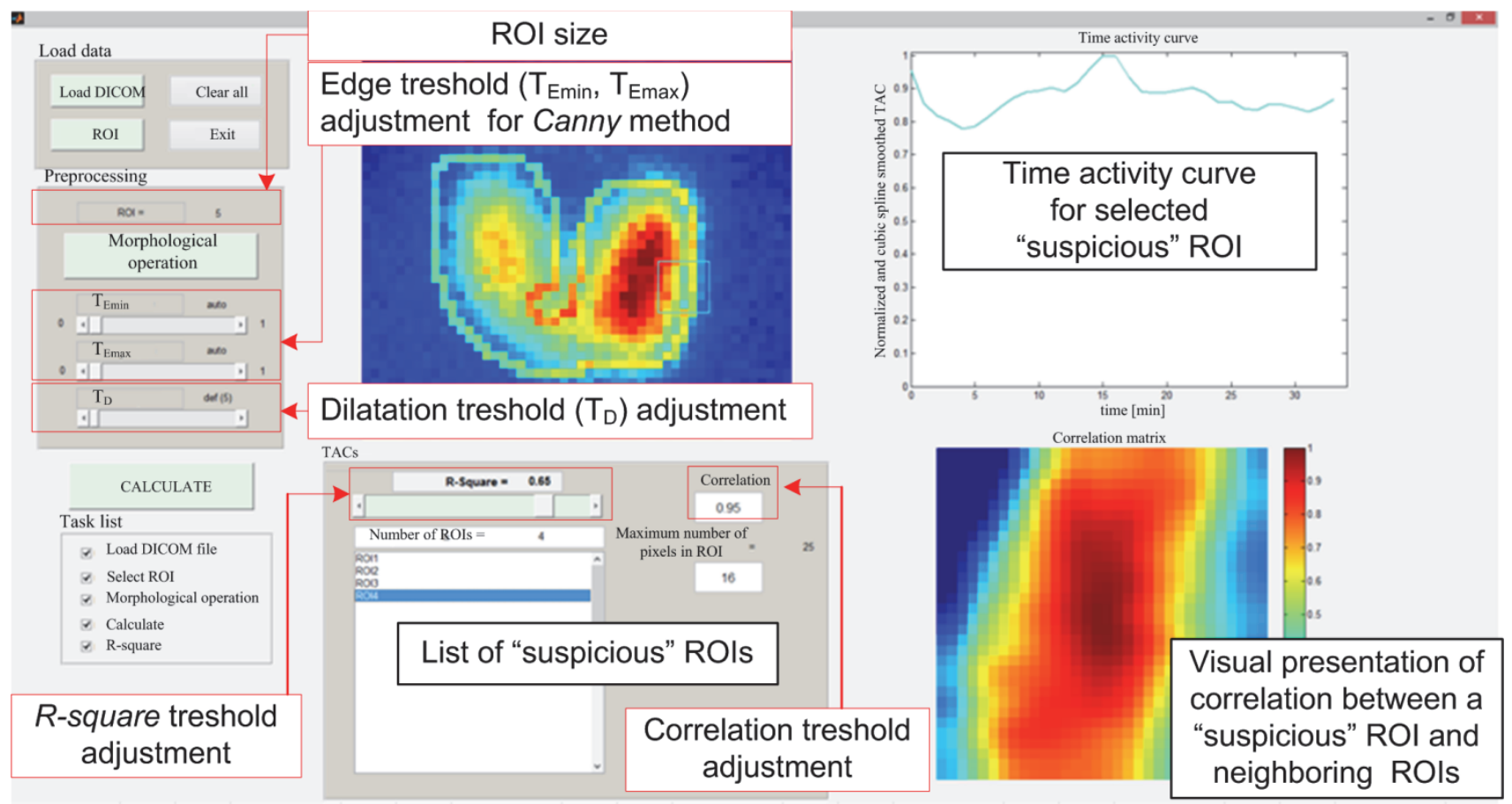

Fig. 4. Print-screen of the Matlab graphical user interface (GUI).

TABLE 1: RESULTS OF APPLIED METHODS FOR LOCALIZATION OF PARATHYROID TUMORS. C - CONVENTIONAL METHOD, $\mathrm{C}+\mathrm{P}-$ CONVENTIONAL METHOD MODIFIED BY POTASSIUM PERCHLORATE, L - SURGERY LOCALIZATION, $\mathrm{V}$ - TUMOR VOLUME, $\mathrm{S}$ - SUBMARINE LOCALIZATION, T - CONCOMITANT THYROID TUMOR, LS - LEFT SUPERIOR, RS - RIGHT SUPERIOR, LI - LEFT INFERIOR, RI - RIGHT INFERIOR.

\begin{tabular}{|c|c|c|c|c|c|c|}
\hline$I D$ & $C$ & $C+P$ & $L$ & $\begin{array}{c}V \\
{\left[\mathrm{~mm}^{3}\right]}\end{array}$ & $S$ & $T$ \\
\hline 1 & + & + & LS & 600 & LS & + \\
\hline 2 & + & + & RI & 1000 & RI & - \\
\hline 3 & + & + & RI & 1632 & RI & - \\
\hline 4 & - & + & LS & 1125 & LS, LI & - \\
\hline 5 & + & + & LI, LS & 600 & LS & + \\
\hline 6 & - & + & RI & 792 & RI & + \\
\hline 7 & + & + & LI & 20 & LI, LS & + \\
\hline 8 & + & + & LI & 2250 & LI & + \\
\hline 9 & - & + & LS & 1008 & LS & - \\
\hline 10 & + & + & LI & 90 & LI & - \\
\hline 11 & - & + & RI & 192 & RI & + \\
\hline 12 & + & + & RI & 560 & RI & + \\
\hline 13 & + & + & LI & 36 & LI & + \\
\hline 14 & + & + & LS & 800 & LS & - \\
\hline 15 & + & + & $\mathrm{RS}$ & 15 & RS, RI & + \\
\hline 16 & - & + & LI & 189 & LI & + \\
\hline 17 & - & + & LS & 912 & LS & - \\
\hline 18 & + & + & $\mathrm{RS}$ & 384 & $\mathrm{RS}$ & - \\
\hline 19 & - & - & $\mathrm{RS}$ & 1296 & $\mathrm{RS}$ & + \\
\hline 20 & + & + & LI & 24 & LI & + \\
\hline
\end{tabular}

If ROIs that are "suspected" to belong to lesion, lie next to each other, it indicates that the lesion size is larger than the size of selected ROI and the procedure should be repeated with a larger ROI size. An example of such a case is presented in Fig. 5. ROI 1 has strong, uniform correlation with neighboring ROIs, but the correlation matrix corresponding to ROI 2 is indicating the boundary of PT lesion.

The presented method successfully localizes parathyroid lesions in case where the subtraction protocol with delayed scans gave negative or unclear results. In these cases, following the standard protocol, it was necessary to repeat the scintigraphic acquisition with additional intravenous potassium perchlorate injection, which extended the time of diagnosis and increased the consumption of radiopharmaceuticals. The presented algorithm proved to be effective in these cases. However, false positive results are expected in case of noisy TACs (in patients that had previous thyroidectomy or in case of patient movement during the study). Also, the described algorithm is still operator dependant due to setting of threshold values.

\section{CONCLUSION}

In this paper we have presented a Matlab interface that enables the implementation and evaluation of new algorithms for PT localization. This application allows flexible analysis of the influence of algorithm parameters on the PT localization results.

We have proposed an efficient method for automatic extraction of thyroid region from the background which represents an initial step in the process of automation of 
PT localization using the Submarine method. The application of developed Matlab interface is illustrated through the example of using the algorithm for PT localization and visualization proposed in [10], [11].

Further research will be focused on the development of a new algorithm that could fully automate the Submarine method. Presented Matlab application and the algorithm for the extraction of thyroid region is the main tool for further development.

(a)
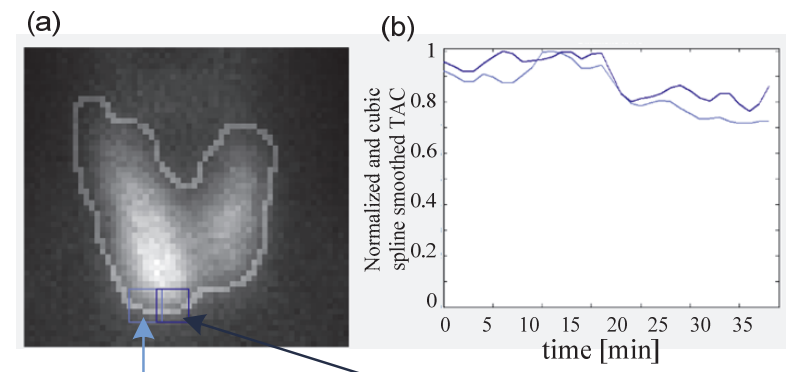

(c)
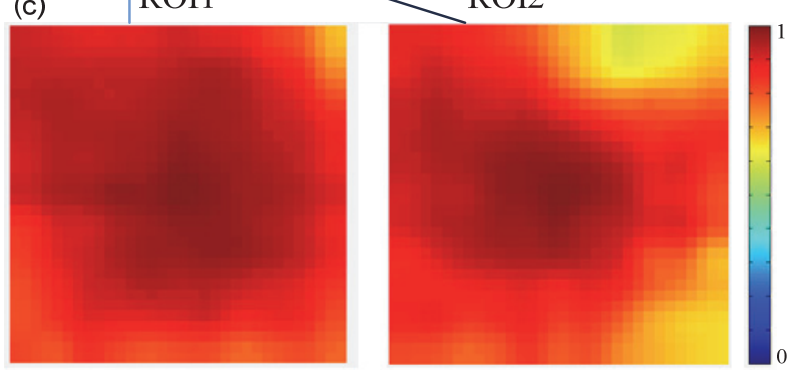

Fig. 5. Illustration of correlation matrix of the two neighboring ROIs over the PT lesion: (a) selected ROIs,

(b) corresponding time-activity curves (TACs),

(c) corresponding correlation matrices.

\section{ACKNOWLEDGEMENTS}

Authors are grateful to Professor Dejan Popović, full member of SASA, School of Electrical Engineering, University of Belgrade, for suggestions during the writing of this paper.

\section{REFERENCES}

[1] M. Giuliano, S. A. Gulec, D. Rubello, G. Boni, M. Puccini, M. R. Pelizzo, G. Manca, D. Casara, G. Sotti, P. Erba, D. Volterrani, A. E. Giuliano, "Preoperative localization and radioguided parathyroid surgery," J Nucl Med, vol. 44, no. 9, pp. 1443-1458, Sept 2003.

[2] E. Hindie, O. Ugur, D. Fuster, M. O'Doherty, G. Grassetto, P Urena, A. Kettle, S. A. Gulec, F. Pons, D. Rubello, "2009 EANM parathyroid guidelines," Eur J Nucl Med Mol Imaging, vol. 36, no. 7, pp. 1201-1216, July 2009.

[3] A. Franceschi, R. Matthews, G. Samara, M. Marzouk, D. Franceschi, "Localizing parathyroid adenoma with Tc-99m sestamibi comparing planar imaging, single photon emission computed tomography (SPECT), and SPECT combined with high quality computed tomography (SPECT-CT)," J Nucl Med, vol. 56, supplement 3, pp. 203-203, 2015.

[4] S. I. Heiba, M. Jiang, J. Rivera, E. Genden, W. Inabnet III, J. Machac, L. Kostakoglu, "Direct Comparison of Neck Pinhole Dual-Tracer and Dual-Phase MIBI Accuracies With and Without SPECT/CT for Parathyroid Adenoma Detection and Localization," Clin nucl med, vol. 40, no. 6, pp. 476-482, 2015.

[5] C. N. Patel, H. M. Salahudeen, M. Lansdown,A. F. Scarsbrook, "Clinical utility of ultrasound and 99mTc sestamibi SPECT/CT for preoperative localization of parathyroid adenoma in patients with primary hyperparathyroidism," Clin Radiol, vol. 65, no. 4, pp. 27887, April 2010.

[6] Y. I. Kim, Y. H. Jung, K. T Hwang, H. Y. Le, "Efficacy of ${ }^{99} \mathrm{mTc}$ sestamibi SPECT/CT for minimally invasive parathyroidectomy: comparative study with ${ }^{99} \mathrm{mTc}$-sestamibi scintigraphy, SPECT, US and CT," Ann Nucl Med, vol. 26, no. 10, pp. 804-810, December 2012.

[7] D. Taieb , E. Hindie, G. Grassetto, P. M. Colletti, D. Rubello, "Parathyroid scintigraphy: when, how, and why? A concise systematic review," Clin Nucl Med, vol. 37, no. 6, pp. 568-74, June 2012.

[8] K. J Nichols, M. B Tomas, G. G Tronco, C. J Palestro, "Sestamibi parathyroid scintigraphy in multigland disease," Nucl Med Commun, vol. 33, no. 1, pp. 43-50, Jan 2012.

[9] A. Koljević Marković, M. M. Janković, I. Marković, G. Pupić, R. Džodić, A. B. Delaloye, "Parathyroid dual tracer subtraction scintigraphy: small regions method for quantitative assessment of parathyroid adenoma uptake," Ann Nucl Med, 2014, in press, doi: 10.1007/s12149-014-0867-0.

[10] M. M Janković., V. Miler Jerković, A. Koljević Marković, D. B Popović, "Algoritam za procenu raspodele radiofarmaka u malim lezijama na dinamskim scintigrafskim snimcima," in Proc. of 58. ETRAN, Zbornik radova, Vrnjačka Banja, 2-5 Jun, ME 1.1, 1-4, 2014.

[11] M. M Janković., V. Miler Jerković, A. Koljević Marković, D. B Popović, "Algorithm for uptake assessment in small lesions based on dynamic scintigraphy scans," Facta Universitatis, accepted for publication.

[12] M. Đurović, "Razvoj algoritma za automatsku dijagnostiku paratiroidnih adenoma," University of Belgrade - School of Electrical Engineering, master thesis, 2014.

[13] J. S. Fleming, R. W. Kenny, "A comparison of techniques for the filtering of noise in the renogram," Phys Med Biol, vol. 22, no. 2, pp. 359-364, Mar 1977.

[14] J. Canny, "A Computational Approach to Edge Detection," IEEE Trans Pattern Anal Mach Intell, vol. PAMI-8, no. 6, pp. 679-698, Nov. 1986. 